ANNALES

POLONICI MATHEMATICI

XLV (1985)

\title{
Über Integraltransformationen, die analytische Funktionen in Lösungen elliptischer Differentialgleichungssysteme überführen
}

von Heinrich Renelt (Halle an der Saale)

\begin{abstract}
It is shown that for every linear uniformly elliptic system $f_{\bar{z}}=v f_{z}+\mu \bar{f}_{z}$ (under suitable assumptions on $v, \mu$ ) there exists an integral transformation carrying analytic functions into solutions of the corresponding system. The kernels of this integral transformations are obtained as solutions of systems of singular integral equations involving the two-dimensional Hilbert transformation.
\end{abstract}

1. Einleitung. Sei $G$ ein Gebiet der $z$-Ebene $E=E_{z}, z=x+i y$. Zu jedem in $G$ gegebenen linearen Differentialgleichungssystem

$$
f_{\bar{z}}=A f+B \bar{f}
$$

mit gewissen Voraussetzungen an $A, B$ gibt es nach I. N. Vekua (siehe [9]) eine Integraltransformation, die in $G$ (bis auf Pole 1. Ordnung) analytische Funktionen in Lösungen von (1.1) transformiert. In vorliegender Mitteilung sollen entsprechende Integraltransformationen für lineare gleichmässig elliptische Differentialgleichungssysteme

$$
f_{\bar{z}}=v f_{z}+\mu \bar{f}_{z}
$$

hergeleitet werden. Wie sich zeigen wird, ergeben sich die Kerne dieser Integraltransformationen als Lösungen von Systemen singulärer Integralgleichungen mit einem komplexen Paramcter (siehe 3.5 unten).

Für Integraloperatoren in Spezialfällen der sogenannten $\Sigma$-monogenen Funktionen vgl. [2], [1], S. $122 \mathrm{ff}$ und [6], S. $163 \mathrm{ff}$. Für Integraltransformationen, die Lösungen des Beltramisystems $g_{\bar{z}}-v g_{z}=0$ in Lösungen des Systems $f_{\bar{z}}-v f_{\bar{z}}=A f+B \bar{f}$ überführen (und eine gewisse Verallgemeinerung hiervon auf höhere Dimensionen) siehe [4].

Der Kürze halber beschränke ich mich auf Koeffizienten $v, \mu$ mit kompakten Trägern und (hauptsächlich) auf beschränkte Gebiete $G$.

2. Bezeichnungen und Hauptergebnis $B_{p}(G)$ die Menge der im beschränkten Gebiet $G$ stetigen Fudktionen $f(z)$ mit verallgemeinerten $A b$ -

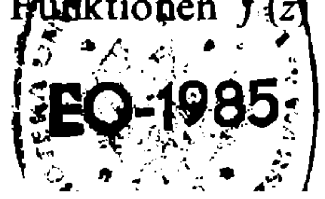


leitungen $f_{z} \in L_{p}^{\text {loc }}(G)$ mit $p>2, A(G)$ sei die Menge der in $G$ analytischen Funktionen. $v(z), \mu(z)$ seien $\forall z$ definierte (Lebesgue-) messbare Funktionen mit

$$
|v(z)|+|\mu(z)| \leqslant k<1 \quad \forall z, \quad \operatorname{supp} v \cup \operatorname{supp} \mu=\Re \subset G .
$$

Bekanntlich bildet der Operator

$$
P_{G} h(z)=-\frac{1}{\pi} \int_{G} \frac{h(t)}{t-z} d \sigma_{t}
$$

( $d \sigma_{t}$ das „Flächenelement” in der $t$-Ebene) den Raum $L_{p}(G)$ mit $p>2$ in den Raum der hölderstetigen Funktionen, die zu $L_{p}(G)$ gehörige Ableitungen nach $z$ und $\bar{z}$ besitzen, ab, die zweidimensionale Hilberttransformation

$$
T_{G} h(z)=-\frac{1}{\pi} \int_{G} \frac{h(t)}{(t-z)^{2}} d \sigma_{t}
$$

bildet $L_{p}(G)$ stetig auf sich ab für jedes (feste) $p>1$, und es gilt

$$
\left(P_{G} h(z)\right)_{z}=T_{G} h(z), \quad\left(P_{G} h(z)\right)_{\bar{z}}=h(z)
$$

fast überall in $G$ und $=0$ fast überall sonst.

Für $f \in B_{p}(G)$ sei

$$
V f(z)=f(z)-P_{G}\left(v f_{z}+\mu \bar{f}_{z}\right)(z) .
$$

Sei weiter $\mathscr{A}(G)=\mathfrak{A}(G, v, \mu)$ die Menge aller $f \in \bigcup_{p>2} B_{p}(G)$, die (zusätzlich verallgemeinerte Ableitungen nach $\bar{z}$ besitzen und die) Lösungen von (1.2) in $G$ sind. Bekanntlich (siehe auch 3. unten) ist $V$ eine umkehrbar eindeutige Abbildung von $B_{p}(G)$ auf sich für alle $p>2$, für die $C_{p} k<1$ ist, wobei $C_{p}$ die Norm von $T=T_{E}$ in $L_{p}=L_{p}(E)$ bedeutet. Dabei gibt es wegen der Stetigkeit von $C_{p}$ in $p$ und $C_{2}=1$ zu jedem positiven $k<1$ ein positives $\varepsilon_{0}$ $(<1)$, so dass $C_{p} k<1$ is $\forall p$ aus dem abgeschlossenen Intervall $\left[2-\varepsilon_{0}\right.$, $\left.2+\varepsilon_{0}\right]$. Ausserdem ist $V$ eine umkehrbar eindeutige Abbildung von $\mathfrak{A}(G)$ auf $A(G)$ (siehe [5]), und es ist $\mathfrak{U}(G) \subset B_{p}(G)$ für jedes $p$ mit $2<p \leqslant 2+\varepsilon_{0}$. Es soll nun bewiesen werden der folgende

SATZ. (I) Die messbaren Funktionen $v, \mu$ mögen die Bedingung (2.1) erfüllen. Dann gibt es zu diesen $v, \mu$ zwei Funktionen $\Phi_{1}(t, z), \Phi_{2}(t, z)$, die für jedes beliebige feste $z$ als Funktionen von $t z u L_{q}=L_{q}(E)$ gehören $\forall q$ mit $1 \leqslant q<2$ und kompakte Träger $\subseteq \mathcal{A}$ besitzen und die durch

$$
U g(z)=g(z)+\frac{1}{\pi} \int_{G}\left\{\Phi_{1}(t, z) g_{t}+\Phi_{2}(t, z) \bar{g}_{t}\right\} d \sigma_{t}
$$


für $g \in B_{p}(G), 2<p \leqslant 2+\varepsilon_{0}$, eine Transformation dieser $B_{p}(G)$ auf sich definieren, die invers $z u V$ ist, $d$. $h$.

$$
U(V f)=f, \quad V(U g)=g,
$$

$\forall f, g \in B_{p}(G)$, jedes $p$ mit $2<p \leqslant 2+\varepsilon_{0}$ und jedes beschränkte Gebiet $G$ mit $\boldsymbol{R} \subset \boldsymbol{G}$.

(II) Wenn $g \in B_{p}(G)$ ( $p, G$ wie unter (I)) auf einer offenen Teilmenge $\omega \subseteq G$ analytisch ist, so ist $U g$ Lösung von (1.2) in $\omega$. Insbesondere ist $U$ eine umkehrbar eindeutige Abbildung von $A(G)$ auf $\mathfrak{U}(G)$.

(III) Sei $h \in L_{p}^{\text {loc }}(G)$ mit $p>2, G \supset \Re$. Dann ist $\int_{G} \Phi_{i}(t, z) h(t) d \sigma_{t}, i=1,2$, analytisch in $z \forall z \in E \backslash \Re$ und hat in $\propto$ eine Nullstelle mindestens 1. Ordnung.

(IV) Sei $g$ analytisch in einem (nicht notwendig beschränkten) Gebiet $\Omega \supset G$ ( $G$ wie unter (I)) bis auf isolierte Singularitäten, sei $S$ die Menge der Singularitäten von $g$ in $\Omega$, und sei $S \subset \Omega \backslash G$. Dann ist $U g$ Lösung von (1.2) in $\Omega \backslash S$, und die Hauptteile der Laurententwicklungen von $g$ und $U g$ stimmen für jeden Punkt aus $S$ überein.

Natürlich gelten entsprechende Aussagen (ohne nennenswerte Änderungen der Beweise) z. B. für den Fall $\Omega \notin G$, aber $v=\mu=0$ ausserhalb von $G$, wobei allerdings dann für die $f \in B_{p}(G)$ nicht nur die Zugehörigkeit der verallgemeinerten Ableitungen nach $z$ zu $L_{p}^{\text {loc }}(G)$, sonderen $\mathrm{zu} L_{p}(G)$ gefordert werden muss und die Klassen $A(G), \mathfrak{A}(G)$ analog einzuschränken sind.

Zunächst mögen noch einige einfache Folgerungen aus dem Satz gezogen werden. Sei $g(z)=A z+B, A, B$ (komplexe) Konstanten, $A \neq 0$. Nach (IV) des Satzes wird

$$
U g(z)=A z+B+\frac{1}{\pi} \int\left(\Phi_{1}(t, z) A+\Phi_{2}(t, z) \widehat{A}\right) d \sigma_{t}
$$

eine Lösung von (1.2) in der ganzen $z$-Ebene mit dem Hauptteil $A z$ in der Laurentreihenentwicklung bezüglich $z=\infty$ (Integrale, bei denen kein Integrationsgebiet angegeben ist, sind stets über den gesamten Träger des Integranden zu berechnen). Nach dem Darstellungstheorem in [3] ist dann $U g(z)$ in (2.8) eine schlichte Abbildung der vollen $z$-Ebene, die Lösung von (1.2) ist und die in $\infty$ die Entwicklung

$$
A z+B+\frac{a_{1}}{z}+\frac{a_{2}}{z^{2}}+\ldots
$$

besitzt. Analog ist im Falle $z_{0} \notin \Re, a=$ const $\neq 0$

$$
\frac{a}{z-z_{0}}+\frac{1}{\pi} \int\left\{\Phi_{1}(t, z) \frac{-a}{\left(t-z_{0}\right)^{2}}+\Phi_{2}(t, z) \frac{-\bar{a}}{\left(t-z_{0}\right)^{2}}\right\} d \sigma_{t}
$$

eine in der ganzen Ebene schlichte Lösung von (1.2) mit einem einfachen Pol in $z_{0}$ mit Residuum $a$ und (dann notwendigerwerse einfacher) Nullstelle in $\infty$. 
Ebenso werden durch

$$
\log \left(z-z_{0}\right)+\frac{1}{\pi} \int\left\{\frac{\Phi_{1}(t, z)}{t-z_{0}}+\frac{\Phi_{2}(t, z)}{t-z_{0}}\right\} d \sigma_{t} .
$$

Fundamentallösungen von (1.2) geliefert, solange $z_{0} \notin \Re$ ist. Es erhebt sich natürlich die Frage, ob (2.9) und (2.10) auch gültig bleiben für $z_{0} \in \Re$. Ohne Beweis sei mitgeteilt, dass bei Gleichungssystemen (1.2) mit $|v|=|\mu| \forall z$ (vgl. das 3. Beispiel in 3. unten) und Lipschitzstetigkeit von $v$ und $\mu \forall z$ der Ausdruck (2.9) als Cauchyscher Hauptwert für fast alle $z$ existiert und für fast alle $z$ mit einer schlichten Lösung von (1.2) mit einem verallgemeinerten Pol 1. Ordnung in $z_{0}$ übereinstimmt. Eine analoge Aussage gilt für den Ausdruck (2.10) bei hölderstetigen Koeffizienten $v, \mu$. Die Beweise dieser Aussagen sind etwas mühsam und beruhen im wesentlichen darauf, dass sich unter den genannten Voraussetzungen an $v, \mu$ die singulären Bestandteile gewisser Cauchyscher Hauptwerte explizit ausrechnen lassen. (Ohne Zweifel gelten entsprechende Aussagen auch für die allgemeinsten Systeme (1.2) bei Lipschitz- bzw. Hölderstetigkeit der $v$ und $\mu$.)

3. Beweis des Satzes. Auf Grund bekannter Eigenschaften singulärer Integraltransformationen (siehe z. B. [8], Kap. II, Satz 3), zu denen auch die Hilberttransformation $T$ gehört, gilt

$$
\int_{G} f \cdot T_{G} g d \sigma=\int_{G} g \cdot T_{G} f d \sigma \forall f \in L_{p}(G), \quad g \in L_{q}(G) \text { und } p^{-1}+q^{-1}=1 .
$$

Sei $L_{q}^{c}(G)=\left\{f: f \in L_{q}(G)\right.$, supp $\left.f \subset G\right\}$. Es gilt:

Wenn für zwei Funktionen $h_{1}, h_{2} \in L_{q}^{c}(G)$ und alle $f \in B_{p}(G)$ mit $p^{-1}+$ $+q^{-1}=1$ gilt $\int\left(h_{1}(z) f_{z}+h_{2}(z) f_{z}\right) d \sigma_{z}=0$, so ist $h_{1}(z)=h_{2}(z)=0$ fast überall in $G$.

Der Beweis von (3.2) ergibt sich einfach durch Betrachtung von $f$ und if, und weil zu jeder Funktion $g \in L_{p}(G)$ ein $f \in B_{p}(G)$ existiert mit $f_{z}=g \forall$ $z \in \operatorname{supp} h_{1} \cup \operatorname{supp} h_{2}$, was im wesentlichen aus der 2. Relation in (2.4) folgt.

Angenommen, $\Phi_{1}(t, z)$ und $\Phi_{2}(t, z)$ würden die Forderungen des Satzes erfüllen. Aus (3.2) folgt dann sofort, dass $\Phi_{1}(\cdot, z)$ und $\Phi_{2}(\cdot, z)$ für jedes feste $z \in G$ als Elemente des $L_{q}^{c}(G)$ eindeutig bestimmt sind. Weiterhin genügt es zum Nachweis von $V^{-1}=U$ zu zeigen, dass $U$ linksinvers zu $V$ ist. Aus der 1. Gleichung in (2.7) unter Berücksichtigung von (3.1) folgt dann für jedes $f \in B_{p}(G), 2<p \leqslant 2+\varepsilon_{0}$,

$$
\begin{aligned}
0= & \int\left\{f_{t}\left[\frac{v(t)}{t-z}+\Phi_{1}(t, z)-v(t) T_{G} \Phi_{1}(\cdot, z)(t)-\overline{\mu(t)} \bar{T}_{G} \Phi_{2}(\cdot, z)(t)\right]+\right. \\
& \left.+\bar{f}_{t}\left[\frac{\mu(t)}{t-z}+\Phi_{2}(t, z)-\mu(t) T_{G} \Phi_{1}(\cdot, z)(t)-\overline{v(t)} T_{G} \Phi_{2}(\cdot, z)(t)\right]\right\} d \sigma_{t}
\end{aligned}
$$




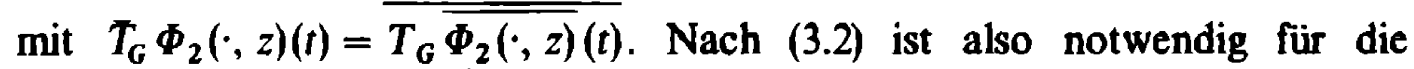
geforderten Eigenschaften der $\Phi_{i}$, dass sie für jedes feste $z \in G$ das durch Nullsetzen der eckigen Klammern in (3.3) entstehende System singulärer Integralgleichungen erfüllen, und hinreichend für die (zunächst unter (I)) geforderten Eigenschaften ist, dass dieses System für jedes $z$ erfüllt ist und die $\Phi_{i}(\cdot, z)$ für jedes feste $z$ zu $L_{q}$ gehören. (Wenn $\Phi_{1}, \Phi_{2}$ Lösungen des genannten Systems sind, so ist natürlich supp $\Phi_{1}(\cdot, z) \cup \operatorname{supp} \Phi_{2}(\cdot, z) \subseteq \Re$ für jedes $z$.)

Sei $\mathfrak{L}_{4}(G)$ der Banachraum der Vektoren $\left(\begin{array}{l}f \\ g\end{array}\right)$ mit $f, g \in L_{4}(G)$ und der Norm $\left(\begin{array}{l}f \\ g\end{array}\right)_{\mathcal{P}_{q}(G)}=\|f\|_{L_{q}(G)}+\|g\|_{L_{q}(G)},\|\cdot\|_{L_{q}(G)}$ die Norm in $L_{q}(G)$. Setzt man

$$
\mathfrak{I}_{v, \mu}\left(\begin{array}{l}
f \\
g
\end{array}\right)=\left(\begin{array}{l}
v T_{G} f+\bar{\mu} T_{G} g \\
\mu T_{G} f+\bar{v} T_{G} g
\end{array}\right)
$$

so lautet das durch Nullsetzen der eckigen Klammern in (3.3) entstehende System

$$
\left(\begin{array}{l}
\Phi_{1}(t, z) \\
\Phi_{2}(t, z)
\end{array}\right)=-\left(\begin{array}{c}
\frac{v(t)}{t-z} \\
\frac{\mu(t)}{t-z}
\end{array}\right)+\mathcal{I}_{v, \mu}\left(\begin{array}{l}
\Phi_{1}(\cdot, z) \\
\Phi_{2}(\cdot, z)
\end{array}\right) .
$$

Für die Norm $\left\|\mathfrak{I}_{v, \mu}\right\|$ von $\mathfrak{I}_{v, \mu}$ in $\mathfrak{I}_{q}(G)$ erhält man sofort die Abschätzung

$$
\left\|\mathfrak{I}_{v, \mu}\right\| \leqslant k^{\prime} \cdot G_{q} \quad \text { mit } \quad k^{\prime}=\|v\|_{\infty}+\|\mu\|_{\infty}, \quad\|\cdot\|_{\infty} \text { die Norm in } L_{\infty} .
$$

Betrachten wir zunächst den Fall $k^{\prime}<1$. Ohne Beschränkung der Allgemeinheit sei das im 2. Abschnitt genannte $\varepsilon_{0}>0$ in diesem Fall noch so gewählt, dass auch gilt

$$
k^{\prime} \cdot C_{q}<1 \forall q \in\left[2-\varepsilon_{0}, 2+\varepsilon_{0}\right] .
$$

Wegen $1 /(t-z) \in L_{q}(G) \forall q<2$ und auf Grund des Banachschen Fixpunktsatzes besitzt (3.5) eine eindeutig bestimmte Lösung in $\mathfrak{L}_{q}(G)$ für jedes $q$ mit $2-\varepsilon_{0} \leqslant q<2$, und diese Lösung ergibt sich mittels der für diese $q$ stark konvergierenden Neumannschen Reihe für $\mathfrak{T}_{v, \mu}$ :

$$
\left(\begin{array}{l}
\Phi_{1}(\cdot, z) \\
\Phi_{2}(\cdot, z)
\end{array}\right)=\sum_{n=0}^{\infty} \mathfrak{I}_{v, \mu}^{n}\left(\begin{array}{l}
f \\
g
\end{array}\right)
$$

mit $f(t)=-v(t) /(t-z), g(t)=-\mu(t) /(t-z)$. Wegen Hölderscher Ungleichung und der Beschränktheit von $G$ ist dann natürlich

$$
\Phi_{i}(\cdot, z) \in L_{q}^{c}(G) \forall q \quad \text { mit } \quad 1 \leqslant q<2, i=1,2 .
$$

Betrachten wir einige Beispiele. 
1. Im Beltramifall $\mu \equiv 0$ folgt $k^{\prime}<1$ aus (2.1). Das System (3.5) reduziert sich in diesem Fall auf die Gleichungen

$$
\Phi_{2}(\cdot, z) \equiv 0, \quad \Phi_{1}(t, z)=-v(t) /(t-z)+v(t) T_{G} \Phi_{1}(\cdot, z)(t) .
$$

2. Im „Antibeltramifall” $v \equiv 0$ folgt ebenfalls $k^{\prime}<1$ aus (2.1). Das System (3.5) lautet dann

$$
\left(\begin{array}{l}
\Phi_{1}(t, z) \\
\Phi_{2}(t, z)
\end{array}\right)=\left(\begin{array}{c}
\bar{\mu} \bar{T}_{G} \Phi_{2}(\cdot, z)(t) \\
-\mu(t))(t-z)+\mu(t) T_{G}\left(\bar{\mu} \bar{T}_{G} \Phi_{2}(\cdot, z)\right)(t)
\end{array}\right) .
$$

3. Auch in der folgenden durchaus bemerkenswerten Klasse von Differentialgleichungssystemen reduziert sich das System (3.5) im wesentlichen auf eine einzige Gleichung. Sei nämlich $|v|=|\mu| \forall z$. Dann gibt es zwei Funktionen $x_{1}, x_{2}$, wobei $\left|x_{2}\right|=$ const $\neq 0 \forall z$ angenommen werden kann, so dass gilt $v=x_{1} \cdot x_{2}, \mu=x_{1} \cdot \bar{x}_{2}$. (Geometrisch sind diese Systeme dadurch gekennzeichnet, dass bei Abbildungen durch Lösungen solcher Systeme die Hauptachsenverhältnisse gewisser infinitesimaler Ellipsenscharen invariant bleiben.) Die Bedingung $k^{\prime}<1$ ist auch hier eine Folgerung aus (2.1), so dass die eindeutige Lösbarkeit des Systems (3.5) durch den bisherigen Stand der Dinge gesichert ist. Aus dem Gleichungssystem (3.5) liest man dann sofort ab

$$
\Phi_{1}(t, z) / \varkappa_{2}(t)=\Phi_{2}(t, z) / \overline{\varkappa_{2}(t)} \equiv \Phi(t, z),
$$

wobei $\Phi(t, z)$ Lösung der singulären Integralgleichung

$$
\Phi(t, z)=\frac{-\chi_{1}(t)}{t-z}-\frac{1}{\pi} \int \Phi(s, z) 2 \operatorname{Re} \frac{\chi_{2}(s) \varkappa_{1}(t)}{(s-t)^{2}} d \sigma_{s}
$$

ist. In diesem Falle ist dann (vgl. [7], 2. Abschnitt)

$$
U g(z)=g(z)+\frac{1}{\pi} \int \Phi(t, z) 2 \operatorname{Re}\left[\chi_{2}(t) g_{t}(t)\right] d \sigma_{t} .
$$

Sei nun zwar (2.1) erfüllt, aber $k^{\prime} \geqslant 1$. Sei $\lambda$ ein reeller Parameter, und anstelle von $v, \mu$ werde in (1.2), (2.5), (3.3), (3.4) stets $\lambda v, \lambda \mu$ gesetzt ((2.1) bleibt unverändert). Dann gilt $\mathfrak{I}_{\lambda v, \lambda \mu}=\lambda \mathfrak{I}_{v, \mu}$, und (3.5) lautet nun

$$
\left(\begin{array}{l}
\Phi_{1}(t, z, \lambda) \\
\Phi_{2}(t, z, \lambda)
\end{array}\right)=\lambda\left(\begin{array}{l}
f \\
g
\end{array}\right)+\lambda I_{v, \mu}\left(\begin{array}{l}
\Phi_{1}(\cdot, z, \lambda) \\
\Phi_{2}(\cdot, z, \lambda)
\end{array}\right)
$$

mit $f, g$ wie in (3.8). Für $|\lambda|<1 / k^{\prime}$ ist $\lambda \mathfrak{I}_{v, \mu}$ kontrahierend in $\mathfrak{I}_{q}(G)$ für genügend nahe bei 2 liegendes $q$, und folglich existiert eine eindeutig bestimmte Lösung, für die sich mittels Neumannscher Reihe bei reellem $\lambda$ die Gestalt

$$
\Phi_{1}(t, z, \lambda)=\sum_{n=1}^{x} \lambda^{n} \cdot \Phi_{1 n}(t, z), \quad \Phi_{2}(t, z, \lambda)=\sum_{n=1}^{x} \lambda^{n} \cdot \Phi_{2 n}(t, z)
$$


mit $\Phi_{\text {in }}(t, z) \in L_{q}(G) \forall q<2$ ergibt, wobei für $|\lambda|<1 / k^{\prime}, \lambda$ reell, diese Reihen stark in $L_{q}(\cdot)$ für jedes $z$ konvergieren. Dabei hängen die $\Phi_{i n}(t, z)$ nicht von $\lambda \mathrm{ab}$, und es gilt supp $\Phi_{\text {in }}(\cdot, z) \subseteq \Re, n=1,2, \ldots, i=1,2$. Somit gilt also für $f=V_{\lambda} g \equiv g-P_{G}\left(\lambda \nu g_{z}+\lambda \mu \bar{g}_{z}\right)$ die Beziehung

$$
g(z)=f(z)+\frac{1}{\pi} \sum_{n=1}^{x} \lambda^{n} \int\left\{\Phi_{1 n}(t, z) f_{t}+\Phi_{2 n}(t, z) f_{t}\right\} d \sigma_{t} .
$$

Andererseits ist natürlich $f=V_{\lambda} g$ für jedes $\lambda$ mit $|\lambda| \leqslant 1<1 / k$ bei gegebenem $f \in B_{p}(G), 2<p \leqslant 2+\varepsilon_{0}$, nach $g$ eindeutig aufiösbar, nämlich es ist auf Grund von (2.4)

$$
g=f+P_{G}(\lambda v h+\lambda \mu \bar{h}),
$$

wobei $h$ Lösung der Gleichung

$$
h=f_{z}+\lambda T_{v \mu} h \quad \text { mit } \quad T_{v \mu} h=T_{G}(v h+\mu h)
$$

ist, die wegen $\left\|T_{v \mu}\right\|_{L_{p}\left(G^{\prime}\right)} \leqslant k C_{p}, G^{\prime}$ ein beliebiges Gebiet mit $\Re \subset G^{\prime} \subseteq G$, für $2<p \leqslant 2+\varepsilon_{0}$ und $|\lambda| \leqslant 1$ ( $\lambda$ reell) mittels der Neumannschen Reihe angegeben werden kann:

$$
h=\sum_{n=0}^{x} \lambda^{n} T_{v \mu}^{n} f_{2} .
$$

Ein Vergleich von (3.12) mit (3.11) und Anwendung des Identitätssatzes für Potenzreihen liefert dann

$$
\int\left\{\Phi_{1 n}(t, z) f_{t}+\Phi_{2 n}(t, z) \bar{f}_{t}\right\} d \sigma_{t}=-\int \frac{v(t) T_{v \mu}^{n-1} \cdot f_{t}+\mu(t) \overline{T_{v \mu}^{n-1} f_{t}}}{t-z} d \sigma_{t}
$$

für jedes $z \in G$ (und dies für jedes beschränkte $G \supset \Re,(3.15)$ gilt also für jedes $z$ ). Bezeichnet man den rechts in (3.15) stehenden Ausdruck mit $H_{n}\left(f_{t}\right)$, so gilt

$$
2 \int \Phi_{1 n}(t, z) f_{t}(t) d \sigma_{t}=H_{n}\left(f_{t}\right)-i H_{n}\left(i f_{t}\right)
$$

Wegen $\left|P_{G^{\prime}} h(z)\right| \leqslant K\left(G^{\prime}, p\right) \cdot\|h\|_{L_{p}\left(G^{\prime}\right)}, K\left(G^{\prime}, p\right)$ eine nur vom Gebiet $G^{\prime}$ und $p>2$ abhängige Konstante, ist

$$
F(h) \equiv \sum_{n=1}^{\infty}\left[H_{n}(h)-i H_{n}(i h)\right]=2 \sum_{n=1}^{\infty} \int \Phi_{1 n}(t, z) h(t) d \sigma_{1}
$$

ein stetiges lineares Funktional auf $L_{p}\left(G^{\prime}\right)$ für jedes $p$ mit $2<p \leqslant 2+\varepsilon_{0}$ und jedes kompakt in $G$ liegende $G^{\prime}$ mit $\Re \subset G^{\prime}$. Auf Grund der Darstellung stetiger linearer Funktionale der $L_{p}$-Räume gibt es also für jedes $z$ ein (wie man noch leicht sieht von den oben genannten $p$ unabhängiges) 
$\Phi_{1}(\cdot, z) \in L_{q}\left(G^{\prime}\right), q=p /(p-1)$, so dass gilt

$$
F(h)=2 \int_{G^{\prime}} \Phi_{1}(t, z) h(t) d \sigma_{t} \forall h \in L_{p}\left(G^{\prime}\right),
$$

d.h. $\sum_{n=1}^{\infty} \Phi_{1 n}(t, z)$ konvergiert für jedes (feste) $z$ schwach in $L_{p}\left(G^{\prime}\right)$ gegen $\Phi_{1}(t, z)$, und wegen supp $\Phi_{1 n}(\cdot, z) \subseteq \mathcal{R} \forall n=2, \ldots$ ist $\Phi_{1}(\cdot, z)$ auch von $G^{\prime}$ unabhängig $\forall G^{\prime}$ mit $\Re \subset \mathrm{G}^{\prime}$, und es ist (nach evtl. Abänderung auf Nullmengen) $\operatorname{supp} \Phi_{1}(\cdot, z) \subseteq \Re$. Entsprechendes gilt für die Reihe $\sum_{n=1}^{x} \Phi_{2 n}(t, z)$ und ihren schwachen Grenzwert $\Phi_{2}(t, z)$.

Wegen (3.12), (3.14), (3.15) und (3.11) für $\lambda=1$ ist also das mit diesen $\Phi_{1}(t, z), \Phi_{2}(t, z)$ gemäss (2.6) gebildete $U$ wieder invers zu $V=V_{1}$ in $B_{p}(G) \forall p$ mit $2<p \leqslant 2+\varepsilon_{0}$ und jedes beschränkte $G \supset \Re$. Damit ist (I) bewiesen. Gleichzeitig ist damit gezeigt:

(3.19) Wenn $v, \mu$ die Bedingung (2.1) erfüllen, so ist das System (3.5) in $L_{q}(E)$ für jedes $q$ mit $\left(2+\varepsilon_{0}\right) /\left(1+\varepsilon_{0}\right) \leqslant q<2$ eindeutig lösbar.

Denn (3.5) war eine notwendige Bedingung für $U=V^{-1}$, also sind die ebengenannten $\Phi_{1}(t, z), \Phi_{2}(t, z)$ Lösung von (3.5), und wegen (3.2) und der Hinlänglichkeit von (3.5) für $U=V^{-1}$ gibt es keine weiteren Lösungen von (3.5).

Die Aussage (II) des Satzes folgt sofort aus (2.7) und der 2. Relation in (2.4). Aussage (III) ergibt sich aus den Beziehungen (3.11)-(3.18) (nachdem man weiss, dass dort überall $\lambda=1$ sein darf) und der Tatsache, dass $P_{G}(\nu h+\mu h)$ ausserhalb von $\mathcal{A}$ eine analytische Funktion mit einer Nullstelle mindestens 1. Ordnung in $\propto$ ist für jedes $h \in L_{p}^{\text {loc }}(G), p>2$. Aussage (IV) folgt aus (I)-(III), indem man Gebiete $G=(\Omega \backslash S) \cap\{|z|<R\}$ mit beliebigem endlichen $R$, das nur genügend gross sein muss, betrachtet.

Bemerkung. Die Vermutung liegt nahe, dass die Norm von $T_{v \mu}$ gleich der Norm von $\mathfrak{I}_{v, \mu}$ ist für alle $p \in\left[2-\varepsilon_{0}, 2+\varepsilon_{0}\right]$ oder dass wenigstens die Reihen (3.10) auch im Falle $k^{\prime} \geqslant 1$ noch für $\lambda=1$ stark in $L_{q}$ konvergieren. Dies muss jedoch hier offen bleiben.

\section{Literatur}

[1] S. Bergmann, Integraloperators in the theory of linear partial differential equations, Ergebn. d. Math. u. ihrer Grenzgebiete, Neue Folge, Heft 23. Berlin, Göttingen, Heidelberg 1961.

[2] L. Bers and A. Gelbart, On a class of differential equations in mechanics of contimua, Quart. Appl. Math. 1 (1943), 168-188.

[3] L. Bers and L. Nirenberg, On a representation theorem for linear elliptic systems with discontimuous coefficients and its applications, Convegno internazionale sulle equazioni lineari alle derivate parziali, Trieste, 25-28 Agosto, 1954, 111-140, Rom 1955. 
[4] B. V. Bojarski, Theorie eines verallgemeinerten analytischen Vektors, Ann. Polon. Math. 17 (1966), 281-320 [Russ.].

[5] -, Verallgemeinerte Lösungen eines Systems von Differentialgleichungen erster Ordnung vom elliptischen Typus mit unstetigen Koeffizienten, Mat. Sb. 43 (1957), 451-503 [Russ.].

[6] G. N. Polożil, Theorie und Anwendung p-analytischer und $(p, q)$-analytischer Funktionem, Kiev 1973 [Russ.].

[7] H. Renelt, Über Extremalprobleme für schlichte Lösungen elliptischer Differentialyleichungssysteme. Comment. Math. Helv. 54 (1979), 17-41.

[8] E. M. Stein, Singular integrals and differentiability properties of functions, Princeton, N. J, 1970 (Russ, Ubersetzung: Moskau 1973).

[9] I. N. Vekua, Systeme von Differentialgleichungen erster Ordnung vom elliptischen Typus und Randwertaufgaben mit einer Anwendung in der Theorie der Schalen, Math. Forschungsberichte II, Berlin 1956 [Úbersetzung aus dem Russ.: Mat. Sb. 31 (1952), 217-314].

MARTIN-LUTHER-UNIVERSITÄT HALLE-WITTENBERG

SEKTION MATHEMATIK

DDR-401 HALLE (SAALE)

UNIVERSITÄTSPLATZ 6

Recu par la Rédaction le 3.05.1979 